UCLA/93/TEP/10

April 1993

hep-ph/9305207

\title{
Disoriented chiral condensate in $(1+1)$ Lorentz-invariant geometry
}

\author{
S.Yu. Khlebnikov 用 \\ Department of Physics, University of California, Los Angeles, CA 90024, USA
}

\begin{abstract}
We consider isospin correlations of pions produced in a relativistic nuclear collision, using an effective theory of the chiral order parameter. Our theory has $(1+1)$ Lorentz invariance as appropriate for the central rapidity region. We argue that in certain regions of space correlations of the chiral order parameter are described by the fixed point of the $(1+1)$ WZNW model. The corresponding anomalous dimension determines scaling of the probability to observe a correlated cluster of pions with the size of this cluster in rapidity. Though the maximal size of clusters for which this scaling is applicable is cut off by pion mass, such clusters can still include sufficiently many particles to make the scaling observable.
\end{abstract}

* On leave of absence from Institute for Nuclear Research of the Academy of Sciences, Moscow 117312 Russia. 
The Centauro events observed in cosmic ray experiments [1] stimulated interest in unusual states of hadronic matter [2]. A now popular idea is that of disoriented

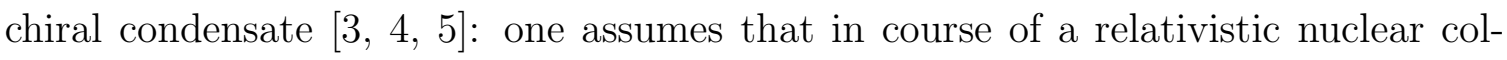
lision, relatively large domains of nuclear matter may emit pions coherently. An experimental signature of such correlated domains would be that certain regions in the angle-rapidity plane are overpopulated by pions of the same isospin, say, there are regions containing mostly $\pi^{ \pm}$'s without many of $\pi^{0}$ s, or vice versa. There is a proposal to look for such events at the SSC 近. These ideas are now discussed not only in connection with leading-particle effects but also for the central rapidity region. Rajagopal and Wilczek [5] consider formation of correlated domains at the secondorder QCD phase transition. They argue that unless temperature drops very fast, the second-order phase transition is unlikely to lead to large correlated domains because the growing correlation length is cutoff by the pion mass. On the other hand, for a sudden quench from high to low temperatures, they find evidence for amplification of long-wavelength pion modes.

The results of ref.[5] are obtained within a (3+1)-dimensional effective theory. One may wonder whether this is always an adequate dimensionality. Indeed, the analysis of experimental data [6, ], in particular those from the CERN SPS collider [8], indicates that the distribution of particles in the central rapidity region possesses $(1+1)$ Lorentz invariance with respect to the longitudinal coordinate $z$ and time $t$. This means that the distribution looks alike in all reference frames obtained from the center-of-mass (c.m.) frame by modest Lorentz boosts in the longitudinal direction. On the other hand, the nuclear matter produced in a collision does not maintain either $(3+1)$ Lorentz invariance or the three-dimensional rotation symmetry. We will argue that in these circumstances, the most long-range part of isospin correlations is described by a $(1+1)$-dimensional effective theory. We also discuss a cutoff role of the pion mass in this case. Our analysis is simpler for the central rapidity region and we concentrate on this region in what follows. This does not exclude a possibility of similar correlation phenomena at larger rapidities.

We need to know statistical weights of various chiral configurations in the regions of physical space producing the central rapidity particles. To maintain the $(1+1)$ Lorentz symmetry, these statistical weights should be determined by a $(1+1)$ Lorentzinvariant effective action. For low-momentum low-frequency modes, the essentially 
unique choice is a variant of the non-linear sigma model. Since we do not have to maintain the full $(3+1)$ Lorentz invariance in this effective theory, more terms become allowed in the chiral lagrangian. In particular, now there are two terms of the second order in derivatives with respect to $z$ and $t$. They form the action

$$
S=\frac{f^{2}}{4} \int d^{4} x \operatorname{Tr}_{\mu} u \partial_{\mu} u^{-1}+g^{2} \int d^{2} x_{\perp} \Gamma_{2}
$$

where $\mu, \nu=z, t, u=\exp \left(i \sum \tau^{a} \pi^{a} / f\right), \pi^{a}$ are the three pion fields, $\tau^{a}$ are the Pauli matrices and $x_{\perp}$ are coordinates in the transverse plane. We will discuss the region of transverse integration below. The quantity $\Gamma_{2}$ is the two-dimensional Wess-Zumino term [9, 10]; it is compatible with both Lorentz and chiral symmetries. For small $\pi^{a}$, the Wess-Zumino term has the form

$$
\Gamma_{2} \approx \frac{1}{6 \pi f^{3}} \int d z d t \epsilon^{\mu \nu} \epsilon^{a b c} \pi^{a} \partial_{\mu} \pi^{b} \partial_{\nu} \pi^{c}
$$

In addition to (回), there are terms containing derivatives with respect to $x_{\perp}$; the one lowest in derivatives is

$$
\frac{1}{4} \int d^{4} x K_{\alpha \beta} \operatorname{Tr} \partial_{\alpha} u \partial_{\beta} u^{-1}
$$

where $\alpha, \beta=x_{\perp 1}, x_{\perp 2}$ and $K_{\alpha \beta}$ is some $2 \times 2$ tensor. Note that due to the $(1+1)$ Lorentz invariance, there are no second-order in derivatives terms that would mix longitudinal and transverse indices.

Because we want to study multiparticle, and in this sense long-range, correlations, we are interested in the values of the couplings $f, g$ and $K_{\alpha \beta}$ attained after an appropriate renormalization. A suitable renormalization procedure in our case is the scaling $z \rightarrow \lambda z$ and $t \rightarrow \lambda t$ leaving the transverse coordinates $x_{\perp}$ unchanged. Note that the longitudinal and transverse couplings can renormalize differently. By simple counting of dimensions, the transverse coupling $K_{\alpha \beta}$ is important when $K_{\alpha \beta} \gtrsim \mu^{2}$, where $\mu$ is a normalization mass. At $K_{\alpha \beta} \ll \mu^{2}$ the scaling laws of the longitudinal theory (1) that describes the $(1+1)$-dimensional fluctuations renormalizing the couplings, are those of the $(1+1)$ WZNW model (see below). The chiral field $u$ scales as $u \propto \mu^{-\gamma / 2}$; for $\gamma$ we take the level $k=1$ value $\gamma=1$. The effective coupling $K(\mu)$ then scales as $\mu^{\gamma}$ and therefore decreases in infrared. Since $K$ decreases slower than $\mu^{2}$, at sufficiently small $\mu$ the dimensionless ratio $K / \mu^{2}$ becomes large and renormalization becomes

\footnotetext{
$\dagger$ Unlike ref. [5], we do not use a linear sigma model because we expect our system to be disordered by fluctuations of the phases of the order parameter, rather than of its magnitude.
} 
complicated. In the real world, however, the accessible values of $\mu$ are bounded from below by the mass of pion. We will see that the renormalized value of pion mass to be used in our case is $m \approx 73 \mathrm{MeV}$. The renormalized value of $K$ at $\mu=m$ is

$$
K_{\alpha \beta}(m)=K_{\alpha \beta}(\Lambda)\left(\frac{m}{\Lambda}\right)^{\gamma}=(26 \mathrm{MeV})^{2} \delta_{\alpha \beta},
$$

where we assume that the cutoff is $\Lambda=1 \mathrm{GeV}$, and $K_{\alpha \beta}(\Lambda)=f_{\pi}^{2} \delta_{\alpha \beta}, f_{\pi}=95 \mathrm{MeV}$. Since $K(m) \ll m^{2}$, we may approximate the behavior of the system at longitudinal distances smaller than $m^{-1}$ by the theory at the fixed point $K_{\alpha \beta}=0$.

At $K_{\alpha \beta}=0$ fluctuations of the order parameter in different regions of the transverse plane decouple from each other and form infinitely many one-dimensional systems. It turns out that long-range correlations in rapidity develop only in some regions of the transverse plane and only for a particular transverse size. We call this favorable size $d$ and will estimate it shortly to be $d \approx 0.8 \mathrm{fm}$. Correlations for other transverse sizes are short-ranged. The size $d$ has the significance of an infrared fixed point: coarse graining in proper time aligns the order parameter in certain transverse regions over the transverse size $d$. Integrating eq.(1) over a transverse region of this size gives a $(1+1)$-dimensional theory

$$
S=\frac{1}{2 \lambda^{2}} \int d z d t \operatorname{Tr}_{\mu} u \partial_{\mu} u^{-1}+k \Gamma_{2} .
$$

This is the WZNW model [9, 10]. Here $\lambda=\sqrt{2} /(f d)$ is a dimensionless coupling and $k$ is an apriori quantized, integer coefficient. Let us temporarily neglect the mass effects. The theory (5) has an infrared stable fixed point at $\lambda^{2}=4 \pi /|k|$ [10, 11]. This gives a value of the transverse scale $d$ for which the rapidity correlations are most long-ranged,

$$
d=\left(\frac{|k|}{2 \pi}\right)^{1 / 2} \frac{1}{f} .
$$

There may be some dependence of $f$ and $d$ on the energy but it need not be dramatic. For estimates, we take $f=f_{\pi}=95 \mathrm{MeV}$. We now have to determine the integer $k$.

The actual presence of the Wess-Zumino term in the effective theory (1), though allowed by the symmetries we discussed so far, is not at all obvious. This term breaks $G$-parity of pions, so it cannot happen in a system consisting of pions alone. In the real system, however, there are other degrees of freedom, and $G$-parity can be broken in interactions of pions with them. For quantized coefficients like $k$, the effects of heavier particles are not necessarily suppressed by inverse powers of their 
masses. The next lowest degree of freedom in nuclear matter are kaons. In the $(3+1)$ dimensional $S U(3)$ (that is including kaons) chiral lagrangian, $G$-parity of pions is broken by the $(3+1)$ Wess-Zumino term [12]. For small fields, the $(3+1)$ Wess-Zumino action has the form

$$
N_{c} \Gamma_{4} \approx \frac{N_{c}}{240 \pi^{2}} \int d^{4} x \epsilon^{\mu \nu \rho \sigma} \operatorname{Tr} \Pi \partial_{\mu} \Pi \partial_{\nu} \Pi \partial_{\rho} \Pi \partial_{\sigma} \Pi
$$

where $N_{c}=3$ is the number of colors, and the $3 \times 3$ matrix $\Pi$ is a parametrization of an $S U(3)$ matrix $U, U=\exp (i \Pi)$. We may write $\Pi=a+A$ where $a$ includes only pions and $A$ includes only kaons and $\eta$. Eq.(7) plays the role of a microscopic action with respect to our effective theory. We have to make a reduction of eq.(7) to the fewer fields and fewer dimensions. This means that we need a portion of eq.(7) where $z$ and $t$ derivatives are applied only to pions but not to kaons or $\eta$. When the transverse integration is restricted to a correlated region of size $d$, the required portion of $\Gamma_{4}$ factorizes,

$$
\Gamma_{4} \rightarrow-\frac{1}{4} \Gamma_{2} Q
$$

where $\Gamma_{2}$ is the $(1+1)$ Wess-Zumino term (2) and

$$
Q \approx-\frac{i}{2 \pi} \int d^{2} x_{\perp} \epsilon^{\alpha \beta} \operatorname{Tr} P \partial_{\alpha} A \partial_{\beta} A
$$

$P$ being a projector $3 \times 3$ matrix, $P=\operatorname{diag}(1,1,0)$. The $\eta$ field does not contribute to eq.(9), so the matrix $A$ in this equation may be regarded as made purely of kaons. Kaons span the projective space $S U(3) /(S U(2) \times U(1)) \approx C P^{2}$, and $Q$ is the integral over the correlated region of the topological density of mappings from the transverse plane into $C P^{2}$ [13]. Eq.(9) is its small-field limit. Therefore, the $(3+1)$ Wess-Zumino action produces a non-zero $(1+1)$ Wess-Zumino action only in the regions where the transverse dependence of kaon fields is topologically non-trivial. These topologically non-trivial configurations of kaons need not be stable; we assume that they can live sufficiently long to give rise to observable effects. Since the integration in eq.(9) is over a finite transverse region, $Q$ need not be integer. The minimal value of $Q$ for which our dimensional reduction is consistent is $Q= \pm 4 / 3$, corresponding to $k=\mp 1$

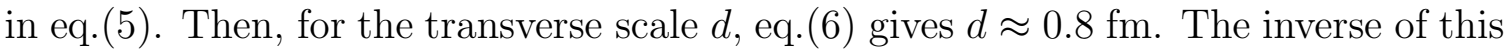
determines a typical transverse momentum of pions produced in correlated regions, $d^{-1} \sim 240 \mathrm{MeV}$.

At the critical point, that is for the favorable transverse size, the longitudinal correlations in the theory (5) are scale-invariant. The (unnormalized) probability 
$P\left(z, z^{\prime} ; t, t^{\prime}\right)$ to have the order parameter $u$ aligned over a distance from $z$ to $z^{\prime}$ for a time from $t$ to $t^{\prime}$ is proportional to a two-point correlation function,

$$
P\left(z, z^{\prime} ; t, t^{\prime}\right) \propto\left\langle u(z, t) u^{-1}\left(z^{\prime}, t^{\prime}\right)\right\rangle \propto\left[\left(z-z^{\prime}\right)^{2}-\left(t-t^{\prime}\right)^{2}\right]^{-\gamma / 2} .
$$

The anomalous dimension $\gamma$ is known [14], $\gamma=3 /(k+2)$; for $k=1, \gamma=1$. We are interested in the probability (10) at the value of proper time $\tau=\tau_{1}$ at which the one-dimensional expansion changes into three-dimensional and the system rapidly hadronizes. This time is proportional to nuclear radius; for nuclei of $\mathrm{Pb}$ or $\mathrm{U}$, Bjorken [6] estimates it to be $\tau_{1} \sim 7 \mathrm{fm} / c$. Going over to the proper time $\tau$ and rapidity $y$ via $z=\tau \sinh y$ and $t=\tau \cosh y$, setting $\tau=\tau^{\prime}=\tau_{1}$, and assuming $y-y^{\prime} \ll 1$, we get

$$
P\left(y, y^{\prime}\right) \propto \frac{1}{\left|y-y^{\prime}\right|^{\gamma}} .
$$

Eq.(11) gives a distribution in rapidity sizes for clusters of pions with a given neutralto-charged ratio, as long as the mass effects are neglected.

In actual comparison of eq.(11) with experimental data, to separate the correlation predicted by (11) from statistical fluctuations, one has to select high-multiplicity events that have clusters of pions with a far from average neutral-to-charged ratio. For example, one selects clusters with more than 90 or less than 5 percent of $\pi^{0}$ 's. Then, one makes a histogram of occurrences of such clusters in all of the selected events versus their sizes in rapidity. As will be seen below, for very large clusters, the probability is cut off by mass effects. However, for clusters whose extent in rapidity is smaller than a certain $\Delta y$, the histogram should show the scaling behavior of probability (11) with the universal exponent $\gamma$.

Now let us consider the role of pion mass and the possibility to observe correlations eq.(11) in the real world. The mass cutoff factor now appearing in eq.(11) is

$$
\exp \left(-m\left[\left(z-z^{\prime}\right)^{2}-\left(t-t^{\prime}\right)^{2}\right]^{1 / 2}\right) \approx \exp \left(-m \tau_{1}\left|y-y^{\prime}\right|\right) .
$$

The renormalized value of pion mass is determined from the scaling equation

$$
m^{2}(m)=m^{2}(\Lambda)\left(\frac{m(m)}{\Lambda}\right)^{\gamma / 2} .
$$

For $\Lambda=1 \mathrm{GeV}, m(\Lambda)=140 \mathrm{MeV}, \gamma=1$, we find $m=73 \mathrm{MeV}$.

Eq.(12) shows that the longer is the one-dimensional evolution of the system, the smaller is the maximal size of clusters for which the scaling law (11) can be observed,

$$
\Delta y \lesssim\left(m \tau_{1}\right)^{-1}
$$


(assuming $\Delta y \lesssim 1$ ). This conclusion itself is not specific for our particular $(1+1)$ dimensional theory; it holds whenever the $(1+1)$ Lorentz invariance is preserved. It is also not unsimilar to the conclusion reached by Rajagopal and Wilczek [5] in the three-dimensional case if we identify the time $\tau_{1}$ as the "cooling" time of the system. There is, however, an important distinction between the thermalized system of ref. [5] and the present case. If the system is thermalized, the number of particles produced by the correlation volume is essentially independent of the energy of the initial colliding particles. On the other hand, the number of particles in a fixed rapidity interval is known experimentally to grow with energy. Selecting high-energy events with large multiplicities per unit rapidity, one may be able to observe correlations (11) even if the interval (14) is relatively small (see below). Increasing the atomic number of colliding nuclei, on the other hand, does not necessarily enhance correlations; in fact, it may even suppress them. Indeed, in our theory, a correlated domain is characterized by the fixed transverse size $d \sim 0.8 \mathrm{fm}$. According to estimates based on the hydrodynamic approach [6], multiplicity per unit rapidity grows with the atomic number $A$ proportionally to the total transverse area, $d N / d y \propto A^{2 / 3}$. Hence, the portion of $d N / d y$ produced by the correlated transverse region is independent of $A$. At the same time, $\tau_{1} \propto A^{1 / 3}$, so the number of correlated particles in the rapidity interval (14) decreases as $A^{-1 / 3}$. In these circumstances, the best place to observe the correlations (11) is high-energy nucleon-nucleon collisions (SSC), rather than nuclei-nuclei collisions. One should keep in mind, however, that using different estimates for the dependence of $d N / d y$ on $A$ existing in the literature [3] may change this conclusion. For hadron-hadron collisions, $\tau_{1} \sim 1 \mathrm{fm} / c$, so the correlations extend over the rapidity interval $\Delta y \sim 2.2$. It seems sufficient to have 15 -20 particles per unit rapidity to be able to observe these correlations and deduce the scaling exponent.

To summarize, we suggest that hadronic matter created in relativistic nuclear collisions is best described not by an effective thermal theory but by an effective theory possessing (1+1) Lorentz invariance. The basis for this suggestion is the observed Lorentz symmetry of the distribution of particles in the central rapidity region. We argue that in certain regions of space correlations of the chiral order parameter are described by the fixed point of the WZNW model. The corresponding anomalous dimension determines the scaling of the probability to observe an isospin-correlated cluster of pions with the size of this cluster in rapidity. Though the maximal size of clusters for which this scaling is applicable is cut off by pion mass, such clusters can 
still include sufficiently many particles to make the scaling observable.

I am grateful to R. D. Peccei for getting me interested in the subject and for discussions and criticism, to S. Chakravarty for discussions on critical fluctuations and renormalization group, and to K. Aoki, P. van Driel, B. Gradwohl and M. von Ins for discussions on various other topics related to this paper. The author is supported by the Julian Schwinger fellowship at UCLA.

\section{References}

[1] C. M. G. Lattes, Y. Fujimoto and S. Hasegawa, Phys. Rep. 65, 151 (1980).

[2] J. Bjorken and L. McLerran, Phys. Rev. D20, 2353 (1979).

[3] A. A. Anselm and M. G. Ryskin, Phys. Lett. B266, 482 (1991); J.-P. Blaizot and A. Krzywicki, Phys. Rev. D46, 246 (1992).

[4] J. D. Bjorken, Int. J. Mod. Phys. A7, 4189 (1992); Acta Physica Polonica B23, 561 (1992); K. L. Kowalski and C. C. Taylor, Case Western Reserve University preprint 92-6, hep-ph/9211282 (1992).

[5] K. Rajagopal and F. Wilczek, Princeton preprints PUPT-1347, IASSNS-HEP92/60, hep-ph/9210253 (1992) and PUPT-1389, IASSNS-HEP-93/16, hepph/9303281 (1993).

[6] J. D. Bjorken, Phys. Rev. D27, 140 (1983).

[7] K. Kajantie and L. McLerran, Nucl. Phys. B214, 261 (1983).

[8] UA5 Collaboration, Phys. Rep. 154, 247 (1987).

[9] S. P. Novikov, Usp. Mat. Nauk, 37, 3 (1982).

[10] E. Witten, Comm. Math. Phys. 92, 455 (1984).

[11] A. M. Polyakov and P. B. Wiegmann, Phys. Lett. 141B, 223 (1984).

[12] J. Wess and B. Zumino, Phys. Lett. 37B, 95 (1971); E. Witten, Nucl. Phys. B223, 422 (1983). 
[13] A. D'Adda, M. Lüscher and P. Di Vecchia, Nucl. Phys. B146, 63 (1978); V. Golo and A. M. Perelomov, Phys. Lett. 79B, 112 (1978); A. J. MacFarlane, Phys. Lett. 82B, 239 (1979).

[14] V. G. Knizhnik and A. B. Zamolodchikov, Nucl. Phys. B247, 83 (1984). 